\title{
Neural Network Applied in City Evaluation
}

\author{
Zhangwei Yang ${ }^{\text {a }}$, Hairong Li, Qing Li \\ Chongqing University of Science \& Technology, Chongqing, China \\ a4858391@qq.com
}

Keywords: Neural network; Comprehensive evaluation; City development.

\begin{abstract}
This paper analyzes the methods of city security evaluation, constructs city community development quality evaluation index system By applying the theory of neural network into the comprehensive evaluation of city development level ,and the comprehensive analysis of city community quality, evaluates on time scales city community quality and its trend, provides theoretical basis for city security evaluation and management. And finally gives the application examples.
\end{abstract}

\section{Introduction}

City is a region of information center and transportation hub with politic, economy, culture, education science and technology, its development can often determine the regional economic development pattern and the spatial distribution of the allocation of resources. How to use varieties of effective ways to the comprehensive evaluation and master urban development trend, becomes very important point. Professor Wang Zongjun, puts forward the fuzzy evaluation method with a set of multi-objective and multi-level theory based on the theory of fuzzy of city comprehensive development level. But this method is lack of self-learning ability, and can't get away from the decision-making process of randomness and evaluation experts subjective uncertainty and fuzziness of knowledge. Neural network theory is applied to evaluate the city development level, provides theoretical basis for city security evaluation and management.

\section{Neural network characteristic}

Neural network is a dynamic system with directed the graph topology structure, it reacts and completes the information processing through a variety of input information, which has dynamic processing and self-learning, self-organizing, adaptive and nonlinear characteristics .Radial Basis Function (RBF) neural network is a feed-forward network with good performance, and it can decide the appropriate network topology based on different issues, with a high approximation precision, a small-scale of network training, fast learning speed and non-existence of local minima problems . The structure of RBF neural network consists of three layers: the input layer, hidden layer and output layer. The structure of topology is showed in Fig1.

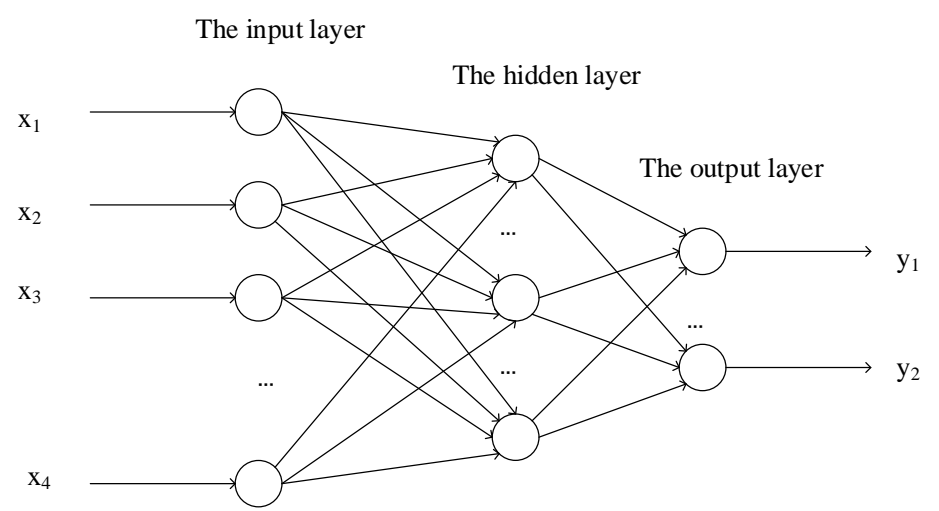

Fig1. RBF neural network structure 
These nodes of input layer are only responsible for passing the input signals to the hidden layer, which including a group of non-linear radial basis function. Gaussian function is generally used as radial basis function, processing the input signal received with nonlinear transformation, and then transmitting the processed signal to the output layer. Gaussian function formula is given by

$$
\varphi_{j}=\exp \left(-\frac{\left\|X-C_{j}\right\|}{2 \sigma_{j}^{2}}\right), j=1,2, \ldots \ldots, \mathrm{L}
$$

Where $\varphi_{\mathrm{j}}$ is the output of the hidden layer, $X \in R^{n}$ is the input of neural network, $\mathrm{C}_{\mathrm{j}}$ is the center of Gaussian function, $\sigma_{j}$ is the width of Gaussian function. $L$ is the number of nodes in the hidden layer.

The output layer processes signals which have been processed by the hidden layer with linear weighted combination. Eventually, the predicted value that is obtained through the neural network processing is one-dimensional vector $\mathrm{y}_{\mathrm{j}}$ :

$$
y_{i}=\sum_{j=1}^{L} w_{i j} \varphi_{j}, j=12, \ldots \ldots, \mathrm{L}
$$

Where $\mathrm{w}_{\mathrm{ij}}$ is the connection weight between the $\mathrm{j}^{\text {th }}$ output of hidden layer and the $\mathrm{i}^{\text {th }}$ neuron of output layer.

\section{City development level comprehensive evaluation Based on neural network}

City community is a relative concept. With the development of society and the development of science technology, its connotation and practice will be constantly developed and improved, there will be many new elements into the city community. In the exploration of urban city community in the process of the evaluation index system, we uses the neural network, concluded that the three levels of evaluation index system of city community, select specific indicators, identify a specific reference index.

There are many kinds of urban city community evaluation models, a commonly used model is: hierarchical analysis model; Pressure-state-response model, "Social economic environment" three elements model, the hierarchical analysis model is used in this paper to integrated use of pressure-state-response model, urban city community quality evaluation index system can be divided into four levels: firstly, the goal layer, namely to urban city community quality as the general objective, comprehensive analysis and characterization of urban city community quality present situation and development trend; The second layer is the essential factor layer, taking the city community pressure, urban city status, social and economic as three elements, evaluating from different aspects about city city community quality; The third layer is the project, in the urban city community system contains some evaluation and analysis on the basic structure for the project, will refine the second of three elements, evaluation of urban city community quality from different angles; The fourth layer as an index, district respectively different urban city community indicators, in the form of a quantitative evaluation of urban city community quality.

\section{Neural network index system}

For the building index system, we can use neural Network and entropy weight to determine the index weight of subjective and objective information respectively, comprehensive weights are obtained, in order to improve the accuracy and credibility of evaluation results.

When determining our security system coordinated integrated evaluation system of the index weight $j$, we should comprehensively consider the results of subjective and objective weights way, that electricity security system coordinated weight value $j$ of comprehensive is concluded.

The determination method of comprehensive weight. 
If we determine weight set by the neural network $A=\left\{a_{1}, a_{2}, \cdots, a_{n}\right\}$, and weight set by entropy weight method: $W=\left\{\omega_{1}, \omega_{2}, \cdots, \omega_{n}\right\}$, the power of city system coordination in the comprehensive evaluation system of the first comprehensive weights of indicators $j$ is as follows:

$$
Z_{j}=\frac{a_{j} \omega_{j}}{\sum_{j=1}^{n} a_{j} \omega_{j}}(j=1,2, \cdots, n)
$$

Calculate comprehensive evaluation index. Through calculation results of the comprehensive evaluation index system and index weight, we use the weighted average model to calculate the comprehensive evaluation index of power ecosystem coordinate $\left(H_{3 e}\right)$, and evaluate the power coordination degree of ecosystem. Its computation formula is as follows:

$$
H_{3 e}(i)=\sum_{j=1}^{n} Z_{j} X_{i j}^{\prime}(i)\left(0 \leq H_{3 e}(i) \leq 1\right)
$$

Index normalized processing. Since the dimension and the order of the magnitude of each indicator has certain differences, we have to eliminate the influences of different dimensions on the evaluation result, so it is necessary to standardize various indicators.

$$
x_{i j}^{\prime}=\left\{\begin{array}{l}
\frac{x_{j}-x_{\min }}{x_{\max }-x_{\min }}-A \\
\frac{x_{\max }-x_{j}}{x_{\max }-x_{\min }}-B
\end{array}\right.
$$

Index after normalization treatment, the matrix proportion is as follows:

$$
Y=\left\{\frac{x_{i j}^{\prime}}{\sum_{i=1}^{m} x_{i j}^{\prime}}\right\}(0 \leq i \leq m, 0 \leq j \leq n)
$$

Calculate index information entropy and information utility value. The first item index $j$ of information entropy value is:

$$
e_{j}=-k \sum_{i=1}^{m} y_{i j} \ln y_{i j}(k=1 / \ln m)
$$

Information utility value depends on the difference in value between 1and the index of information entropy $e_{j}$. Its computation formula is:

$$
d j=1-e_{i}
$$

Calculate entropy of index. The entropy value of the $j$ item parameter values is defined as weight:

$$
\omega_{j}=\frac{d_{j}}{\sum_{i=1}^{m} d_{j}}
$$

\section{Application Examples}

Choosing a set of cities \{Tianjin, Harbin, xi 'an, Zhengzhou, Shijiazhuang, Tangshan\} from a set of comprehensive evaluation decision support system. Table 1 shows the evaluation principles, using fuzzy comprehensive evaluation method and neural network comprehensive evaluation method, two groups of results are obtained

Table 1.Evaluation principles

\begin{tabular}{l|c|c|c|c|c|c|} 
city & Tianjin & Harbin & Xi'an & Zhengzhou & Shijiazhuang & Tangshan \\
\hline
\end{tabular}




\begin{tabular}{c|c|c|c|c|c|c}
\hline $\begin{array}{c}\text { Fuzzy comprehensive } \\
\text { evaluation results }\end{array}$ & 0.334 & 0.328 & 0.277 & 0.290 & 0.320 & 0.255 \\
\hline $\begin{array}{c}\text { Neural network } \\
\text { evaluation results }\end{array}$ & 0.349 & 0.316 & 0.271 & 0.283 & 0.328 & 0.273 \\
\hline
\end{tabular}

\section{Conclusion}

Using neural network knowledge learning, synthetic evaluation of city development level and the network for comprehensive evaluation to the development level of other cities is completely feasible. When the sample model is more close to the evaluation results, it is more accurate to evaluate the development of the city status.

\section{Reference}

[1] Zhang Biao, Study on assessment index of the livable city [J]. China Population, Recourse and Environment, 2005, 15 (87) p: 70-73.

[2] Yin Gongfeng. Artificial neural network information processing principle. Pattern recognition and artificial intelligence.2000.vol.3 .No.1. p: l -13.

[3] Hong Jihua, Song Yilan. Application research of analytic hieararchy process neural networkin water environmental planning [J]. Environmental Science \& Technology, 2000, 23(1) p32-36.

[4] Chang Tao. Comprehensive Evaluation Indicator System of City Community [D]. Dalian: Dalian University of Technology, 2003.

[5] Zhou Jian-fei, On uncertainties of indicating system for eco-environmental evaluation of residential communities [J]. Journal of Safety and Environment, 2005, 5(2) p.24-27. 